\title{
Una vivencia de gestión ambiental en el marco del proyecto: mejora de la oferta educativa en gestión ambiental rural, en centros educativos de Guápiles y Sarapiquí
}

\section{An Environmental Management Experience Within the Project: Improvement of Educational Opportunities in Rural Environmental Management in Sarapiqui and Guapiles' Schools}

\author{
Giselle León León ${ }^{l}$ \\ División de Educología \\ Centro de Investigación y Docencia en Educación (CIDE) \\ Universidad Nacional, Costa Rica \\ Heredia, Costa Rica \\ leongiselle@hotmail.com
}

Recibido 09 de abril de 2010 • Aceptado 24 de junio de 2010

\begin{abstract}
Resumen. Un aspecto de suma importancia para la educación mundial y en particular para la educación costarricense es la gestión ambiental como estrategia educacional, por lo que a continuación se describe la vivencia del proyecto Mejora de la oferta educativa en gestión ambiental rural (MOE-GAR) en su primer etapa 2009, cuya población estuvo conformada por docentes de primaria y secundaria de diferentes áreas de las Direcciones Regionales Educativas de Guápiles y Sarapiquí de Costa Rica.

El proyecto se desarrolló a lo largo del ciclo lectivo de 2009, mediante un trabajo conjunto entre los docentes involucrados, diferentes instituciones gubernamentales y no gubernamentales, Dio como resultado un proceso de construcción de conocimientos en gestión ambiental, a partir del cual se plantearon y desarrollaron propuestas en los diferentes centros educativos.
\end{abstract}

Palabras clave. Gestión ambiental, instituciones educativas, vivencias.

Abstract. A very important aspect of worldwide education and, particularly for Costa Rican education, is the environmental management as an educational strategy. For this reason, this article describes the experience of the project Mejora de la Oferta Educativa en Gestión Ambiental Rural (MOE-GAR) during its first stage in 2009. The population was comprised by teachers from different areas of the rural school districts of Guapiles and Sarapiquí.

As a part of this project, we worked with teachers, different government and nongovernment institutions, throughout the 2009 school year; obtaining as a result, a process of knowledge development in environmental management. Based on this, several proposals were developed in different educational institutions.

Key words: Environmental management, educational institutions, experience.

\footnotetext{
Máster en Administración Educativa, grado obtenido en el año 2006. Bachiller en la Enseñanza de las Ciencias, Universidad Nacional, Costa Rica (2002). Licenciatura en Ciencias de la Educación con énfasis en Didáctica de las Ciencias (2003). Actualmente académica de la División de Educología y coordinadora por parte de la Universidad Nacional de Costa Rica del proyecto "Mejora de la oferta educativa en gestión ambiental" y profesora de Física de segundaria. Dentro de las últimas publicaciones: Educación para el cambio: protección del planeta, El síndrome de Burnout, una realidad en educación "La gestión ambiental como estrategia educacional" (experiencia del proyecto: Mejora de la oferta educativa en gestión ambiental-Sarapiquí.
} 


\section{Introducción}

La gestión ambiental ha sido una de las preocupaciones de las políticas educativas nacionales y ambientales, razón por la cual se ha incorporado en el proceso educativo, como parte integral la formación de los individuos y de los colectivos.

En el marco del mejoramiento de la calidad de la educación y en particular de la educación ambiental, desde el 2006, el Observatorio del Desarrollo (OdD) de la Universidad de Costa Rica inició el trabajo en centros educativos de la gran área metropolitana, coordinado de manera general por MSc. Álvaro Fernández G. A partir del 2009, la división de Educología del Centro de Investigación y Docencia en Educación (CIDE), Universidad Nacional, se incorporó, para trabajar con poblaciones rurales de la Región Huetar Norte, con centros educativos de primaria y secundaría de las Direcciones Regionales de Sarapiquí y Guápiles ${ }^{2}$. Estuvo financiado por el programa de Regionalización Interuniversitaria Región Huetar Norte $^{3}$ del Consejo Nacional de Rectores de Costa Rica (CONARE).

Este proyecto ofrece, a la comunidad educativa de las poblaciones involucradas, la formación en gestión ambiental de sus profesores, herramientas para emprender acciones concretas en sus comunidades educativas, frente a la problemática ambiental rural, articulando su experiencia educativa, en los centros docentes con su práctica comunitaria más allá de las aulas. De tal manera que se construyan conocimientos, materiales y ejercicios prácticos relacionados con problemas ambientales en el entorno inmediato de los (las) participantes.

Por lo anterior, para los diferentes colaboradores del proyecto, es muy grato presentar a los lectores este documento, que describe el trabajo desarrollado por docentes de las Direcciones Regionales de Sarapiquí y Guápiles (primer ciclo, segundo ciclo, tercer ciclo y educación diversificada), que participaron directamente en gestión ambiental, tanto en el campo conceptual, como en el campo metodológico y de proyección local y regional de la misma, en los $26^{4}$ centros educativos en los cuales estuvo focalizado el proyecto Mejora de la oferta educativa en gestiona ambiental rural (MOE-GAR) en su primera etapa 2009.

\section{Metodología empleada}

La estrategia fundamental del proyecto se centró en el desarrollo de un curso de "Innovación educativa en gestión ambiental" (IE-GA) - de una semana de duración-, tres talleres y seguimiento a lo largo del año. Participaron, como mediadores pedagógicos, las responsables del proyecto en la zona MSc. Guiselle Hidalgo y Bach. Lorena Salmerón, por parte de la Universidad de Costa Rica, y MSc. Giselle León, por la Universidad Nacional. Además de las fuerzas vivas de la comunidad -que tienen conocimiento de la problemática ambiental de la zona-; a partir de este momento el grupo de docentes diseñó y ejecutó, con la comunidad, proyectos ambientales de acuerdo con las necesidades y lectura del contexto.

\footnotetext{
Las Direcciones Regionales Educativas son distribuciones geográficas que hace el Ministerio de Educación Pública, para atender todo el país en materia educativa. Guápiles es el distrito 01 del cantón de Pococí, inscrito a la provincia de Limón, zona Caribe de Costa Rica. Sarapiquí es el cantón número 10 de la provincia de Heredia, zona Norte de Costa Rica.

3 Se debe indicar que Guápiles no corresponde a la Región Huetar Norte, pero se incluyó en el trabajo por el interés mostrado en la temática, además esta iniciativa no solo se desarrolló en esta zona, sino que también se trabajó en San Carlos en coordinación con la UNED y en otras regiones con otros fondos.

4 Seleccionados por los Asesores regionales de Ciencias, tomado como referente el interés y compromiso en el tema.
} 
En la primera parte, en el curso (desarrollado del 28 al 30 de abril del 2009) se analizaron enfoques y experiencias notables de innovación educativa -entendidas estas como las acciones que el docente en su entorno y contacto directo va a generar en colaboración con otros actores que se incorporen a su práctica educativa- en temas relacionados con la gestión ambiental. Es decir, con la intención definida de promover valores positivos hacia el medio (solidaridad, responsabilidad, compromiso, respeto hacia toda forma de vida, convivencia armoniosa, entre otros) se realizaron un conjunto de acciones orientadas a incidir en los elementos del currículo, haciendo uso de elementos educativos curriculares o extracurriculares (Rodríguez, Zúñiga y Guier, 1996).

Las sesiones del curso constaron de experiencias vividas en las instituciones sobre gestión ambiental rural y conferencias impartidas por expertos de diferentes organizaciones comunales (Ministerio de Educación, Ministerio de Salud, líderes comunales, organizaciones no gubernamentales, entre otros), las cuales versaron sobre el respeto a sí mismo y al cuido de toda forma de vida (hacia un enfoque educativo integrado en salud y ambiente, gestión integrada de la salud ambiental para centros educativos, didáctica, entre otros). Además, se desarrollaron procesos de retroalimentación con personas de la comunidad, quienes ya tenían experiencias en los campo de ejecución o con los mismos compañeros docentes que ya trabajaban proyectos de gestión ambiental. Lo anterior, con el fin de que puedan seguir desarrollando acciones concretas en pro del medio ambiente, de manera autónoma y sostenible.

Los docentes participantes (primer ciclo, segundo ciclo, tercer ciclo y educación diversificada) elaboraron, como producto de este curso, dentro del marco de la gestión ambiental, una propuesta acorde con las necesidades de sus centros educativos. La asistencia al curso fue obligatoria, por lo cual se llevó control diario con las respectivas listas de asistencia y su correspondiente firma por cada uno de los participantes. Solo se permitió una ausencia justificada, pues se les dio un certificado reconocido por servicio civil.

En el segundo segmento del trabajo propuesto (abril-diciembre), los docentes ejecutaron, dentro de sus instituciones, los proyectos propuestos en el primer segmento (curso), como complemento a las actividades descritas. Con el objetivo de apoyarles y, a su vez, con el fin de evaluar el avance de las propuestas y registrar las horas de ejecución invertidas por los docentes encargados, durante el año se le dio seguimiento a cada propuesta, mediante una visita mensual por parte del equipo del proyecto y asesores de Ciencias del Ministerio de Educación Publica (MEP) de las direcciones regionales participantes.

Como ya se mencionó, a lo largo del año se ofrecieron tres talleres: el primero sobre manejo de desechos sólidos el 19 de julio, ya que la mayoría de las propuestas se estaban desarrollando sobre este tema; de igual manera, el 25 de septiembre se realizó una actividad de seguimiento con los participantes de los docentes de Sarapiquí, donde uno de los objetivos fue compartir experiencias; los días 15 y 16 de octubre se realizó una gira de campo a Tortuguero, en la cual personal del Ministerio de Ambiente Energía y Telecomunicaciones (MINAET) les impartió charlas, se visitó el centro de acopio de la comunidad, además por la noche se hizo una gira al parque Nacional Tortuguero y, en el segundo día, se expusieron algunos avances de las propuestas. La participación en los talleres fue obligatoria y se acreditó como parte del aprovechamiento del trabajo iniciado en el curso (fase inicial).

Entre los productos, cada participante debió realizar un informe con los objetivos del trabajo desarrollado, actividades cumplidas o en proceso, y conclusiones y proyecciones. Después de todo este proceso, en diciembre, se entregaron los certificados acreditados por servicio civil a los asesores, para que ellos los distribuyeran. 


\section{Elementos conceptuales}

\section{Gestión ambiental}

La gestión ambiental se puede conceptualizar como un “(...) campo que busca equilibrar la demanda de recursos naturales de la Tierra con la capacidad del ambiente natural, debe responder a esas demandas en una base sustentable" (Colby, 1990, p. 1). Su orientación, por lo tanto, va dirigida a la consecución de niveles de calidad ambiental, con un uso sostenible de los recursos económicos, materiales y humanos que requieran dicho fin. La gestión ambiental responde al "cómo hay que hacer" para conseguir lo planteado por el desarrollo sostenible, es decir, para conseguir un equilibrio adecuado para el desarrollo económico, crecimiento de la población, uso racional de los recursos, y protección y conservación del ambiente. Abarca un concepto integrador superior al del manejo ambiental: de esta forma no sólo están las acciones a ejecutarse por la parte operativa, sino también las directrices, lineamientos y políticas formuladas desde los entes rectores, que terminan mediando la implementación.

Este particular se desarrolla, a nivel rural, pues es tan importante generar cambios en gestión ambiental tanto a nivel urbano como rural, ya que por las necesidades -si bien no son las mismasse debe potenciar un manejo integral del desarrollo sostenible a nivel mundial, mismo que sólo se puede generar mediante la educación ambiental.

\section{Educación ambiental}

"Proceso que consiste en reconocer los valores y aclarar conceptos con el objetivo de fomentar las aptitudes y actitudes necesarias para comprender y apreciar las interrelaciones entre el hombre, su cultura y su medio biofísico" (UNESCO, 1970 citado por Garcia y Nando, 2000, p. 48). Tomando como referencia esta definición, se puede indicar que educación ambiental implica una toma de decisiones y de formas de comportamiento; no solo a nivel ambiental, sino en las formas de vida que se pueden construir en el proceso educativo formal y no formal.

\section{Educación ambiental para el desarrollo sostenible}

Durante la Cumbre Mundial para el Desarrollo Sostenible, celebrada en 2002 en Johannesburgo, se reafirmó la necesidad de alcanzar un desarrollo sostenible tal, que solo se puede lograr asumiendo la responsabilidad conjunta de fomentar y fortalecer los tres pilares inseparables de la protección del medio ambiente: el desarrollo social y el desarrollo económico a nivel local, nacional, regional y mundial (Naciones Unidas, Cumbre Mundial para el Desarrollo Sostenible, 2002).

La educación para el desarrollo sostenible implica un cambio potencial en la forma de pensar y actuar, un cambio de paradigma. Se hace necesario un desarrollo económico de forma no tradicional, que busque nuevas vías, nuevos estilos de trabajo, un desarrollo armónico y planificado de forma sostenible en el tiempo para las presentes y futuras generaciones. Se define como un desarrollo duradero. El desarrollo sostenible plantea la formación de una conciencia ciudadana, la formación de una actitud diferente, de una forma nueva de pensar y proceder para afrontar los retos que este desarrollo requiere. Este reto solo es alcanzable, si se enfrenta desde la educación. 


\section{¿Cómo educar para el desarrollo sostenible?}

Ante todo se debe asumir la educación como un proceso permanente, es decir, para toda la vida; que salga de las aulas y se proyecte en las comunidades; que eduque en una actitud de enfrentar a lo mal hecho, a lo que daña al medio ambiente; que sea un proceso planificado y asumido con responsabilidad y con la participación ciudadana a través de las organizaciones sociales.

La educación ambiental para el desarrollo sostenible debe desarrollar, en los ciudadanos, conocimientos, sensibilidad y conciencia sobre el medio ambiente y su sostenibilidad; educar en el espíritu de la conservación, en el amor a sus semejantes, en la tolerancia con las especies y nosotros mismos,; debe preparar, integralmente, y continuamente al ser humano para la vida.

\section{¿Cómo lograr el vínculo con el entorno escolar y comunitario desde los centros educativos?}

Es necesario ofrecer a los educandos conocimientos sobre los elementos medioambientales del entorno escolar y comunitario. Para esto resulta imprescindible que el centro educativo tenga su caracterización ambiental. Esta caracterización se puede ir logrando y ampliando a partir del estudio local (diagnóstico) que deben realizar los docentes y educando, al cumplir el principio del vínculo con la localidad.

\section{La transversalidad en los programas de estudio}

Como se indica en la Política Educativa hacia el siglo XXI "La educación asume el reto ético, que fortalece aquellos valores y actitudes que le den a la propia vida, de lo económico y a lo social un sentido altruista, inspirador, incorruptible y humanista (...) (Costa Rica, Ministerio de Educación Púbica. Consejo Superior de Educación, 1994, p. 5), es decir, debe tomar como gran eje transversal los valores fundamentales del humanismo. De esta manera, el abordaje sistemático de los valores en el currículo nacional pretende potenciar el desarrollo socio afectivo y ético de los estudiantes. A partir del eje transversal de los valores y de las obligaciones asumidas por el estado desde la legislación existente, en Costa Rica se han definido los siguientes temas transversales (Costa Rica, Ministerio de Educación Pública, 2005, p. 2):

Cultura ambiental para el desarrollo sostenible

Educación integral de la sexualidad

Educación para la salud y vivencia de los derechos humanos

Educación para la democracia y la paz

\section{Eje transversal: cultura ambiental para el desarrollo sostenible}

Uno de los ejes tranversales que se fortalece con este proyecto es el de cultura ambiental para el desarrollo sostenible el cual indica: 
La educación ambiental se considera como el instrumento idóneo para la construcción de una cultura ambiental de las personas y las sociedades, en función de alcanzar un desarrollo humano sostenible, mediante un proceso que les permita comprender su interdependencia con el entorno, a partir del conocimiento crítico y reflexivo de la realidad inmediata, tanto biofísica como social, económica, política y cultural.

Tiene como objetivo que, a partir de ese conocimiento y mediante actividades de valoración y respeto, las y los estudiantes se apropien de la realidad, de manera que, la comunidad educativa participe activamente en la detección y solución de problemas, en el ámbito local, pero con visión planetaria. (Costa Rica, MEP, 2005, p. 3)

\section{Innovación educativa}

Según Libedinsky (2001),

(...) las innovaciones didácticas emergentes son propuestas de enseñanza generadas por docentes de aula, caracterizadas por la ruptura y oposición con prácticas vigentes consolidadas, profundamente ensambladas con el contenido curricular disciplinar, y que atienden tanto a los intereses culturales de los docentes que las diseñan y lideran, como a los intereses culturales de sus estudiantes(...). (p. 60)

Lo importante de esta concepción es que son innovaciones-acciones-pensadas por docentes que tienen contacto directo y cotidiano con alumnos: no son institucionales, no son tradicionales, es decir, no son megaproyectos educativos. Por el contrario, poseen claridad a qué práctica tradicional oponerse, no se encuentran desarticuladas respecto del contenido curricular disciplinar, presentan intereses culturales de intersección entre lo que le interesa al docente y al estudiante.

En este proyecto uno de los elementos que se pueden tomar como un referente de innovación es que se pretende involucrar a las fuerzas vivas de la comunidad, a diferentes instituciones públicas como Universidades, MEP, Ministerio de Salud, Ministerio de Ambiente y Energía, organizaciones no gubernamentales (redes de apoyo), etc. Otro aspecto que se puede indicar como innovador lo constituye la exigencia de la participación activa de la comunidad educativa en el entorno próximo. Además, no sólo participan los docentes de Ciencias y es más vivencial que solo desarrollar un curso teórico sobre educación ambiental. Es aprender haciendo. Hacer en el que se les acompaña durante todo el ciclo lectivo.

\section{Manejo de residuos sólidos}

Para iniciar en este tema se deben tener claros los conceptos de basura y desecho. Basura se puede definir como: "Todo residuo sólido o semisólido putrescible o no putrescible, excluyendo las excretas de origen humano o animal. En esta definición se incluyen los desperdicios, desechos, cenizas, elementos del barrido de calles, residuos industriales y comerciales, de establecimientos hospitalarios y de mercados entre otros". Por su parte, residuo sólido es: "Todo objeto, sustancia o elemento en estado sólido o semisólido, que se abandona, bota, rechaza o desprende" (Costa Rica. Presidencia de la República de Costa Rica, 1987, p. 1). 
De las definiciones anteriores se puede indicar que a estos conceptos se les pueden dar diferentes interpretaciones, por lo cual en este documento se va a entender como basura aquel material del cual ya no se pude hacer más uso, por estar contaminado o en mal estado y, por residuo sólido, aquel material al cual se le puede dar más uso, ya sea para el que fue creado u otro según la necesidad del usuario.

Existen muchas clasificaciones de los residuos, por ejemplo, "extraordinarios", que son los que no se generan todos los días, tales como los electrodomésticos, fluorescentes, y los "ordinarios", aquellos que se producen todos los días. Estos se pueden subclasificar en "biodegradables", cuya materia se descompone por la acción que realizan los microorganismos, los hongos, las bacterias y otros; y los "no biodegradables", desechos que se generan, en mayor escala, los que la misma naturaleza no es capaz de degradar o descomponer, tal como los plásticos, los metales, el tetra pack, entre otros.

\section{¿Qué podemos hacer con tantos desechos originados de manera cotidiana en los centros educativos y en el diario vivir?}

Rechazar: evitar comprar productos cuyos materiales o empaques producen un alto grado de contaminación.: bolsas plásticas, estereofón, baterias, entre otros.

Reducir: comprar lo estrictamente necesario, ahorre agua y energía eléctrica.

Reutilizar: utilizar varias veces diferentes implementos que lo permiten, por ejemplo, utilizar envases retornables o reutilizables, y regalar o vender aquellos objetos que ya no se necesiten.

Reciclar: recolectar y clasificar materiales considerados como desechos, con el objeto de que puedan ser procesados por las industrias y vuelvan a entrar en la corriente del consumo. Por lo tanto, cuando se recicla algún material, solo se está realizando una parte de un proceso completo, el cual se centra en su reconversión industrial o artesanal para convertirlo en un objeto igual o parecido para volver a usarse.

Cada ser humano adulto produce casi media tonelada de basura por año; por lo cual, para que esta no cause problemas, se deben tomar medidas que permitan controlarla (Avendaño, 2010).

\section{Importancia de reciclar:}

- Para evitar la contaminación del ambiente: se salvan espacios usados para botar y enterrar basuras. La mayoría son simples vertederos al aire libre, más que rellenos sanitarios. Estos se llenan rápidamente y hay que ampliarlos o buscar otros sitios, ya que son fuentes de contaminantes y enfermedades para su entorno.

- Para evitar la contaminación atmosférica: la quema de basura es una de las principales fuentes de contaminación atmosférica, debido a que los desechos expulsan gases que contribuyen a la lluvia ácida, contienen metales pesados tóxicos y la cancerígena dioxina; además de producir miles de toneladas de cenizas tóxicas que terminan en los vertederos de basura.

- $\quad$ Porque no requiere tanto tiempo.

- Porque es económico: si las personas se organizan resulta más barato separar para reciclar, que pagar todo el proceso de recolección de basura municipal. El gobierno local ahorra recursos que puede utilizar en otras cosas, además de que se puede obtener beneficios económicos directos de la actividad de reciclar. 
- $\quad$ Porque salva materiales y recursos: nuestros recursos son finitos; si no reciclamos, se pueden perder más rápidamente. Se obtiene aluminio reciclando latas de aluminio o a partir de la bauxita, mineral que probablemente se acabará dentro de doscientos años, si no disminuye el actual consumo. Por otra parte, se puede usar periódico para hacer nuevo papel, en lugar de talar árboles para fabricarlo a partir de la pulpa virgen.

Materiales que se pueden reciclar: la mayoría de los materiales que componen los residuos cotidianos se pueden reciclar; unos con más facilidad que otros, dependiendo del precio del momento dado, de los centros de acopio y empresas existentes, de la facilidad de transporte hasta estos centros y del nivel organizativo de las comunidades.

- El vidrio: reusar los envases, exigir envases retornables que puedan ser usados muchas veces, es decir, aplicar el reciclaje. Reciclando vidrio se salva una tonelada de recursos por cada tonelada producida. Debe ser clasificado y depositado por colores. De lo contrario, durante el proceso de fundición se pueden producir mezclas de colores. Los cuellos y tapas metálicas deben ser removidos, porque contaminan el proceso.

- $\quad$ Papel cartón: el papel es un material que se recicla con facilidad. Es conveniente preguntarle al comprador sobre la manera de clasificar el papel y el cartón. Algunas empresas reciben cualquier tipo de papel y cartones mezclados; otras, exigen que se separen en categorías específicas como: cartones, papel de computadora, cartones grises, revistas. En la mayoría de los casos, el cartón parafinado no es reciclable.

- El aluminio: es sencillo de reciclar y adquiere un buen precio en el mercado. Pertenecen a este grupo: la lata, el papel aluminio, la chatarra y el material ferroso. Al reciclarlos, se salvan enormes cantidades de energía y recursos que, por ejemplo, a la naturaleza le costaría de 100 a 500 años en degradar.

- $\quad$ El plástico: el problema de los plástico comienza por su uso inapropiado, pues es un material muy resistente y de larga duración, utilizado para fabricar objetos que tienen un tiempo de uso muy corto, que pronto son arrojados a la basura. Esto se ejemplifica con la diversidad de bolsas, las cuales en la intemperie pueden durar hasta 20 años en degradarse y pueden ser usadas para trasportar algo por menos de cinco minutos, sin nombrar empaques y productos desechables plástico. Vale rescatar que a nivel de Costa Rica, solo se pueden reciclar el plástico uno y dos, y que muchos de los materiales ya lo traen identificado en las partes inferiores de los recipientes (Avendaño, 2010).

\section{Logros del trabajo}

Los mayores logros obtenidos a través del proceso desarrollado durante el 2009 fueron los siguientes:

Se capacitaron 26 centros educativos entre las dos Direcciones Regionales, impactando a los circuitos 01, 02, 03, 04 y 05 de Guápiles y 01, 02, 03 de Sarapiquí.

Se capacitó a 37 docentes de primaria y secundaria en servicio de las dos Direcciones Regionales, con un alcance del $270 \%$, pues inicialmente el proyecto consideró como grupo meta a 10 docentes de secundaria de la Dirección Educativa de Sarapiquí.

Se desarrolló el curso con tres talleres complementarios, y se les dio seguimiento, a lo largo del año, a las todos los docentes involucrados. 
Se logró implementar un $89 \%$ de las propuestas en los centros educativos, donde el $87 \%$ de ellas se desarrolló sobre la sensibilización de la población educativa (educandos, administrativos, docentes padres y madres), en cuanto a separación de desechos sólidos. De tal manera que en un $50 \%$ de las escuelas y colegios se implementaron pequeños centros de acopio, cuyo material fue recogido por diferentes organizaciones locales ya existentes. El restante 2\% trabajó en ornato de la institución, huertas hidropónicas, siembra de árboles, uso racional del agua y elaboración de abonos orgánicos con los desechos generados en la institución.

Se logró la unión de la acción académica, lo cual sin duda brinda beneficios y logros derivados de la articulación entre dos o más Universidades, lo cual permite evidenciar uno de los objetivos de la Comisión Regional Interuniversitaria de Costa Rica (CRI), el cual consiste en unir capacidades de las universidades y de sus académicos en pro del desarrollo local.

\section{Consideraciones finales}

El recorrido por las diferentes puestas en práctica en algunos centros educativos de las Direcciones Regionales de Sarapiquí y Guápiles nos induce a pensar en un aprendizaje necesario para el ambicioso objetivo del proyecto MOE-GAR: contribuir en la construcción de cambios actitudinales con respecto a la gestión ambiental. Si bien la educación no puede resolver por sí misma los problemas ambientales, es imprescindible para desarrollar la conciencia de conservación. Por lo anterior, se pueden indicar las siguientes consideraciones finales:

- Se acompañó en la construcción y puesta en práctica de propuestas y proyectos de educación ambiental existentes e inéditos, atendiendo a la visión de contexto en las comunidades participantes.

- Se conformó un equipo de trabajo interdisciplinario e interinstitucional en las instituciones participantes, lo que permitió desarrollar acciones concretas en gestión ambiental.

- A partir de la creación de nuevos espacios para las relaciones interdisciplinarias y para la proyección de acciones, las comunidades educativas participantes movilizaron las dinámicas internas de las instituciones educativas, las que, desde el conocimiento significativo, se cualificaron y participaron en la solución de problemáticas ambientales particulares.

- Los problemas ambientales son situaciones anormales que ponen en riesgo el equilibrio de las relaciones dinámicas entre naturaleza y sociedad. Por ello, deshacerse indiscriminadamente de basura y desechos de todo tipo en cualquier sitio produce residuos y emanaciones inexistentes antes en la tierra y en la atmósfera. Por ello, el manejo adecuado de los desechos sólidos surge como una salida para disminuir, en la medida de lo posible, los vertederos a cielo abierto. Esta preocupación se evidenció en las comunidades educativas participantes, ya que la mayoría de las instituciones desarrollaron parte de sus propuestas en ese tema.

- La gestión ambiental es un proceso cuya finalidad consiste en propiciar la convivencia entre hombre-naturaleza-sociedad de tal manera que se tomen decisiones y se construyan cambios actitudinales, no sólo a nivel ambiental sino en las propias formas de vida, tanto a nivel formal como informal, para que las generaciones venideras disfruten y conserven toda esa 
variedad de ecosistema con que cuenta el planeta, y donde el amor y el respeto por el medio sea la consigna de todos los seres vivos.

\section{Recomendaciones}

- Se incentiva a los centros educativos participantes, en aras de los resultados hasta ahora alcanzados, a seguir poniendo en práctica la presente gestión ambiental como estrategia educacional comunitaria, previa contextualización, con el propósito de lograr la integración de los factores sociales con los que se ha venido trabajando, pues la práctica demostró que los logros en este sentido son eminentemente satisfactorios.

- $\quad$ Se recomienda a CONARE continuar proyectándose a las comunidades, ya que muchos docentes tienen interés en desarrollar actividades como las dadas en el marco de este proyecto, pero siempre es importante la capacitación y el acompañamiento que puedan dar las Universidades.

- Como parte de los retos del equipo investigador, desarrollar un segundo segmento adecuado a las necesidades expresadas por los docentes, con mayor participación de especialistas locales.

\section{Referencias bibliográficas}

Avendaño, D. (2010, Febrero). Taller de manejó de desechos sólidos. Charla impartida en el recinto del proyecto UNA Campus sostenible. Heredia, Universidad Nacional.

Colby, M. E. (1990). Environmental Management in Development: The Evolution of Paradigms. Washington: World Bank Discussions Papers, número 80.

Costa Rica. Ministerio de Educación Pública. Consejo Superior de Educación. (1994). Política Educativa hacia el siglo. XxI. Recuperado el 11 de enero de 2010, de http:/www.oei.es/quipu/ costarica/politicaeducativasigloXXI.pdf

Costa Rica. Ministerio de Educación Pública. (2005). Programa de estudio Ciencias III Ciclo. Despacho del Viceministro Académico. San José, Costa Rica: Autor.

Costa Rica. Presidencia de la República de Costa Rica. (1987, junio 20). Decreto 19094. Reglamento sobre el manejo de basuras. Recuperado el 29 de marzo de 2010, de http:/www.ccad.ws/ documentos/legislacion/CR/D-19094.pdf

García, G. y Nando, R. (2000). Estrategias didácticas en educación ambiental. España: Editorial ALJIBE.

Libedinsky, M. (2001). La innovación en la Enseñanza: Diseño y documentación de experiencias de aula. Argentina: Editorial Paidós. 
Naciones Unidas. Cumbre mundial sobre el desarrollo sostenible. (2002, 26 de agosto a 4 de septiembre). El compromiso de Johannesburgo por un desarrollo sostenible. Recuperado el 10 de enero de 2010, de http://www.cinu.org.mx/eventos/conferencias/johannesburgo/documentos/ dec johannesburgo.pdf

Rodríguez, M., Zúñiga, M. E., Guier, E. (1996). Ambientalización de cursos de una carrera universitaria. San José, Costa Rica: Editorial EUNED. 